\title{
A characterization of the average tree solution for tree games
}

\author{
Debasis Mishra • A. J. J. Talman
}

Accepted: 27 October 2009 / Published online: 7 November 2009

C The Author(s) 2009. This article is published with open access at Springerlink.com

\begin{abstract}
For the class of tree games, a new solution called the average tree solution has been proposed recently. We provide a characterization of this solution. This characterization underlines an important difference, in terms of symmetric treatment of the agents, between the average tree solution and the Myerson value for the class of tree games.
\end{abstract}

Keywords Tree $\cdot$ Graph games $\cdot$ Myerson value $\cdot$ Shapley value

\section{JEL Classification $\quad$ C71}

\section{Introduction}

In a cooperative game, cooperation is not always possible for all coalitions of agents. The limited possibilities of cooperation can often be represented by an undirected graph or tree in which cooperation is only possible if agents are directly or indirectly connected to each other. The study of graph games was pioneered in Myerson (1977). He proposed as solution concept the Myerson value, which equals the Shapley value of the induced restricted game. Other characterizations of Myerson value are given in Myerson (1980) and Borm et al. (1992). For the subclass of tree games Herings et al. (2008) recently introduced the average tree solution (AT solution), being the average

D. Mishra $(\varangle)$

Planning Unit, Indian Statistical Institute, 7 Shahid Jit Singh Marg, New Delhi 110016, India e-mail: dmishra@isid.ac.in

\section{A. J. J. Talman}

CentER, Department of Econometrics and Operations Research, Tilburg University, P.O. Box 90153 5000 LE Tilburg, The Netherlands

e-mail: talman@uvt.nl 
of the marginal contribution vectors corresponding to all directed spanning trees of the graph. The AT solution is easy to compute and belongs to the core of the game if the game is superadditive, as is proved in Herings et al. (2008) and follows from earlier results in Demange (1994, 2004), Kaneko and Wooders (1982), and Le Breton et al. (1992).

How does the average tree solution differ from the Shapley and Myerson value? We address this question in this paper. We show that for tree games the average tree solution is uniquely characterized by efficiency, dummy, linearity, strong symmetry, and independence in unanimity games. The first three are also used by Shapley (1953) to characterize the Shapley value. The other two axioms replace the symmetry axiom for the Shapley value. Strong symmetry is also satisfied by the Shapley value and requires that if only the grand coalition has non-zero worth, then every agent receives the same payoff. Independence in unanimity games requires that if a player joins a coalition in a unanimity game, then the payoff of any agent in the coalition that is not linked to that player remains the same. While in a unanimity game the Shapley value treats all members of a coalition equally, the AT solution treats each of them in proportion to the number of agents this agent is connected to outside the coalition.

\section{The average tree solution and the Myerson value}

We follow the notations in Herings et al. (2008). A tree game is denoted by $(N, v, L)$, where $N=\{1, \ldots, n\}$ is a finite set of agents, $G=(N, L)$ is a tree, an undirected graph without cycles, on $N$, and $v$ is a characteristic function assigning to each connected subset $S$ of $N$ the worth $v(S)$ of coalition $S$. Only coalitions that are connected in $(N, L)$ are assumed to be able to cooperate. Without loss of generality, it is assumed that the grand coalition $N$ is connected. For a graph $(N, L)$ the collection of connected coalitions in a graph $(N, L)$ is denoted by $C(N, L)$ and, for a subset $T$ of $N, C^{m}\left(T, L_{T}\right)$ denotes the collection of maximally connected subsets or components in the subgraph $\left(T, L_{T}\right)$ of $(N, L)$ restricted to $T$. Finally, $\mathbb{G}$ denotes the class of tree games.

Let $(N, L, v)$ be a tree game. Since $(N, L)$ is a tree, every $i \in N$ induces a unique directed spanning tree, denoted by $T(i)$, with node $i$ being the root. With respect to $T(i), i \in N$, the marginal payoff of player $j \in N$ is defined by

$$
t_{j}^{i}(N, L, v)=v\left(S_{T(i)}(j)\right)-\sum_{k \in F_{T(i)}(j)} v\left(S_{T(i)}(k)\right),
$$

where, for $j \in N, F_{T(i)}(j)$ is the set of nodes that come immediately after $j$ in tree $T(i)$ and $S_{T(i)}(j)$ is the set of nodes consisting of $j$ and all nodes that come after $j$ in tree $T(i)$. The average tree solution is the average of these marginal payoffs over all $n$ directed spanning trees.

Definition 1 For a tree game $(N, L, v) \in \mathbb{G}$ the average tree solution (AT solution) assigns the following payoff to agent $j \in N$ :

$$
A T_{j}(N, L, v)=\frac{1}{n} \sum_{i \in N} t_{j}^{i}(N, L, v) .
$$


The Myerson value (Myerson 1977) is defined as follows. ${ }^{1}$ For a graph game $(N, L, v)$, let the induced restricted game be the cooperative game $\left(N, v^{L}\right)$, where the characteristic function $v^{L}: 2^{n} \rightarrow \mathbb{R}$ is defined as:

$$
v^{L}(S)=\sum_{T \in C^{m}\left(S, L_{S}\right)} v(T) \quad \forall S \subseteq N .
$$

The Myerson value of graph game $(N, L, v)$, denoted as $\mu(N, L, v)$, is the Shapley value of the game $\left(N, v^{L}\right)$, i.e., $\mu(N, L, v)=\operatorname{Sh}\left(N, v^{L}\right)$.

\section{Axioms}

In this section, we state the axioms we use in our characterization of the average tree solution. The objective is to use axioms similar to the ones in Shapley (1953). The first three axioms are standard in the cooperative game literature, while the last two axioms replace the symmetry axiom in Shapley (1953).

Definition 2 An allocation $\pi: \mathbb{G} \rightarrow \mathbb{R}^{n}$ satisfies efficiency if for any game $(N, L, v) \in \mathbb{G}$,

$$
\sum_{i \in N} \pi_{i}(N, L, v)=v(N) .
$$

For $i \in S, S \in C(N, L)$, let the marginal contribution of agent $i$ to coalition $S$ in game $(N, L, v)$ be $\Delta_{i}^{N, L, v}(S)=v(S)-v^{L}(S \backslash\{i\})$.

Definition 3 An allocation $\pi: \mathbb{G} \rightarrow \mathbb{R}^{n}$ satisfies dummy if for any game $(N, L, v) \in \mathbb{G}$ and $i \in N$ it holds that $\pi_{i}(N, L, v)=0$ whenever $\Delta_{i}^{N, L, v}(S)=0$ for all $S \in C(N, L)$ and $S \ni i$.

Definition 4 An allocation $\pi: \mathbb{G} \rightarrow \mathbb{R}^{n}$ satisfies linearity if for any two games $(N, L, v)$ and $(N, L, w)$ in $\mathbb{G}$ and any $a, b \in \mathbb{R}$,

$$
\pi_{i}(N, L, a v+b w)=a \pi_{i}(N, L, v)+b \pi_{i}(N, L, w) \quad \forall i \in N,
$$

where the game $a v+b w$ is defined as $(a v+b w)(S)=a v(S)+b w(S)$ for all $S \in C(N, L)$.

In the cooperative game literature, a standard axiom for the Shapley value is symmetry. However, in trees, agents are typically not in a symmetric position. For example, a leaf is in a different position than a node that is connected to more than one node. Therefore, we replace the symmetry axiom in Shapley (1953) by a weaker version and add a new axiom that reflects the asymmetric position of nodes in a tree.

The next axiom is also satisfied by the Shapley value.

\footnotetext{
1 The Myerson value is defined for any graph game, also for graphs which contains cycles.
} 
Definition 5 An allocation $\pi: \mathbb{G} \rightarrow \mathbb{R}^{n}$ satisfies strong symmetry if for any game $(N, L, v) \in \mathbb{G}$ with $v(S)=0$ for all $S \in C(N, L)$ and $S \neq N$, we have $\pi_{i}(N, L, v)=$ $\pi_{j}(N, L, v)$ for all $i, j \in N$.

The strong symmetry axiom says that if all proper coalitions have worth zero, then every agent receives the same payoff. Together with efficiency this axiom implies that when $v(S)=0$ for all $S \in C(N, L), S \neq N$, each player gets an equal share of $v(N) / n$.

The last axiom is on the class of unanimity tree games. For $T \in C(N, L)$, let $u_{T}(S)=1$ if $T \subset S$ and $u_{T}(S)=0$ otherwise. The triple $\left(N, L, u_{T}\right)$ defines the unanimity tree game for coalition $T$.

To motivate the axiom, we use a result of Herings et al. (2008). Consider any $S \in C(N, L)$. For $j \in S$ we say that agent $j$ represents agent $k \notin S$ for coalition $S$ if on the unique path connecting $j$ to $k$ every agent except agent $j$ is outside $S$. Let $p_{S}^{L}(j)$ be the number of agents that agent $j \in N$ represents for $S$, with $p_{S}^{L}(i)=0$ when $i \notin S$. Herings et al. (2008) show that for any unanimity tree game ( $\left.N, L, u_{T}\right), T \in C(N, L)$, it holds that

$$
A T_{j}\left(N, L, u_{T}\right)= \begin{cases}\frac{1+p_{T}^{L}(j)}{n} & \forall j \in T \\ 0 & \text { otherwise. }\end{cases}
$$

The ratio $\left(1+p_{T}^{L}(j)\right) / n$ reflects the representation power of agent $j \in T$ for coalition $T$ in tree $(N, L)$. The AT solution distributes the payoff in unanimity games according to the representation power of the agents.

On the other hand, the Myerson value for unanimity game $\left(N, L, u_{T}\right), T \in C(N, L)$, is equal to

$$
\mu_{j}\left(N, L, u_{T}\right)= \begin{cases}\frac{1}{|T|} & \forall j \in T \\ 0 & \text { otherwise. }\end{cases}
$$

Thus, in unanimity games the Myerson value treats agents within $T$ equally. We now introduce an axiom that captures this difference in its weakest form.

Definition 6 An allocation $\pi: \mathbb{G} \rightarrow \mathbb{R}^{n}$ satisfies independence in unanimity games if for any game $(N, L, v) \in \mathbb{G}$ and $T, T \cup\{j\} \in C(N, L)$ with $j \in N \backslash T$ it holds that for all $i \in T$ with $\{i, j\} \notin L$

$$
\pi_{i}\left(N, L, u_{T}\right)=\pi_{i}\left(N, L, u_{T \cup\{j\}}\right) .
$$

Independence in unanimity games says that, when an agent joins a coalition, any agent in the coalition not being linked to that agent receives the same payoff. Since the graph is a tree, a player outside any coalition is linked to just one player in that coalition. Only for this player in the coalition the representation power, and therefore the payoff, will change. 


\section{Characterization}

In this section we prove that the average tree solution is uniquely characterized by the axioms stated in the previous section and show that these axioms are independent from each other.

Theorem 1 On the class of tree games, the average tree solution is the unique solution satisfying efficiency, dummy, linearity, strong symmetry, and independence in unanimity games.

Proof It is easy to verify that the average tree solution satisfies all five axioms. Therefore, let $\pi$ be any allocation satisfying all axioms. Consider the unanimity game $\left(N, L, u_{N}\right)$. By efficiency and strong symmetry,

$$
\pi_{k}\left(N, L, u_{N}\right)=\frac{1}{n} \quad \forall k \in N
$$

Since $p_{N}^{L}(j)=0$ for all $j \in N$, we have

$$
A T_{k}\left(N, L, u_{N}\right)=\frac{1}{n} \quad \forall k \in N
$$

Therefore, $\pi\left(N, L, u_{N}\right)=A T\left(N, L, u_{N}\right)$. Take any $1 \leq t<n$ and suppose $\pi\left(N, L, u_{S}\right)=A T\left(N, L, u_{S}\right)$ for all $S \in C(N, L)$ with $|S|>t$. We show that $\pi\left(N, L, u_{T}\right)=A T\left(N, L, u_{T}\right)$ for any $T \in C(N, L)$ with $|T|=t$. Since $N$ is connected and $t<n$, there exists $j \notin T$ such that $T \cup\{j\}$ is connected. Let $S=T \cup\{j\}$. By our induction hypothesis,

$$
\pi_{k}\left(N, L, u_{S}\right)=A T_{k}\left(N, L, u_{S}\right)=\frac{1+p_{S}^{L}(k)}{n} \quad \forall k \in S .
$$

Consider any $k \notin T$. By definition, for every $S \in C(N, L)$ containing $k$, we have $\Delta_{k}^{N, L, u_{T}}(S)=0$. Hence, by the dummy axiom

$$
\pi_{k}\left(N, L, u_{T}\right)=0=A T_{k}\left(N, L, u_{T}\right) .
$$

Let $i$ be the unique agent in $T$ such that $\{i, j\} \in L$. Consider any $k \in T \backslash\{i\}$. By the independence in unanimity games axiom and Eq. 3,

$$
\pi_{k}\left(N, L, u_{T}\right)=\pi_{k}\left(N, L, u_{S}\right)=A T_{k}\left(N, L, u_{S}\right)=\frac{1+p_{S}^{L}(k)}{n} .
$$

We show that $p_{S}^{L}(k)=p_{T}^{L}(k)$. Denote the set of agents who $k$ represents for $S$ as $P_{S}^{L}(k)$. Clearly, $P_{S}^{L}(k) \subseteq P_{T}^{L}(k)$. Assume for contradiction, there exists $k^{\prime} \in P_{T}^{L}(k) \backslash$ $P_{S}^{L}(k)$. Since the graph is cycle-free, there exists a unique path $\left(k, k^{1}, k^{2}, \ldots, k^{q}, k^{\prime}\right)$ in $(N, L)$ such that $k^{1}, \ldots, k^{q} \notin T$. Since $k^{\prime} \notin P_{S}^{L}(k), j \in\left\{k^{1}, \ldots, k^{q}\right\}$. This implies that there is a path $\left(k, k^{1}, \ldots, k^{r}, j\right)$ from $k$ to $j$ such that $k^{1}, \ldots, k^{r} \notin T$. Clearly, 
$r \geq 1$, since $\{k, j\} \notin L$. Since $T \cup\{j\}$ is connected, there also exists in $T$ a path from $k$ to $j$, yielding a contradiction. Hence, Eq. 3 implies that

$$
\pi_{k}\left(N, L, u_{T}\right)=\frac{1+p_{S}^{L}(k)}{n}=\frac{1+p_{T}^{L}(k)}{n}=A T_{k}\left(N, L, u_{T}\right) .
$$

By Eqs. 4 and 6, for every $k \in N, k \neq i$, we have

$$
\pi_{k}\left(N, L, u_{T}\right)=A T_{k}\left(N, L, u_{T}\right) .
$$

By efficiency, we get that $\pi_{i}\left(N, L, u_{T}\right)=A T_{i}\left(N, L, u_{T}\right)$.

Finally, Herings et al. (2008) show that every tree game can be written as a linear function of unanimity tree games. By the linearity axiom, the result then follows.

We now show that the axioms are independent. The Myerson value satisfies efficiency, dummy, strong symmetry, and linearity, but fails independence in unanimity games. This follows from the fact that in any unanimity game $\left(N, L, u_{T}\right)$ the Myerson value assigns every agent $i \in T$ a payoff equal to $\frac{1}{|T|}$.

Equal sharing, where every agent receives $v(N) / n$, satisfies efficiency, strong symmetry, linearity, and independence in unanimity games, but fails dummy.

The zero allocation, where every agent gets zero payoff, satisfies strong symmetry, linearity, dummy, and independence in unanimity games, but fails efficiency.

Next, consider the allocation $\pi^{d}=t^{i}(N, L, v)$ for some $i \in N$. Clearly, $\pi^{d}$ satisfies efficiency, dummy, and linearity. For the unanimity tree game $\left(N, L, u_{S}\right)$, $S \in C(N, L)$, call agent $k \in S$ a dictator in $S$ if in the tree $T(i)$ node $k$ is the first agent on any directed path from $i$ to any other agent in $S$. It is clear that for a dictator agent $k$ in $S, \pi_{k}^{d}\left(N, L, u_{S}\right)=1$ and $\pi_{j}^{d}\left(N, L, u_{S}\right)=0$ if $j \neq k$. Now, consider the unanimity games $\left(N, L, u_{S}\right)$ and $\left(N, L, u_{S \cup\{j\}}\right)$. Let $k \in S$ be such that $\{k, j\} \notin L$. In case agent $k$ is not a dictator in $S$, he is also not a dictator in $S \cup\{j\}$ and in both games his payoff is zero. In case agent $k$ is a dictator in $S, k$ will also come first in any directed path from $i$ to any other node in $S \cup\{j\}$. Hence, $k$ will remain a dictator in $S \cup\{j\}$, and his allocation is one in both games. This shows that $\pi^{d}$ satisfies independence in unanimity games. Now consider a tree game $(N, L, v)$ such that $v(S)=0$ for all $S \in C(N, L)$ and $S \neq N$. In that case, $\pi_{i}^{d}(N, L, v)=t_{i}^{i}(N, L, v)=v(N)$ and $\pi_{j}^{d}(N, L, v)=0$ for all $j \neq i$. Hence, $\pi^{d}$ fails strong symmetry. Thus, allocation $\pi^{d}$ satisfies efficiency, dummy, linearity, and independence in unanimity games, but fails strong symmetry.

Finally, consider for unanimity tree games the average tree solution and for every other tree game the Myerson value. This allocation satisfies efficiency, dummy, independence in unanimity games, and strong symmetry, but fails linearity.

Acknowledgements We thank two anonymous referees for detailed comments about the paper. Stimulating conversations about the average tree solution with Gerard van der Laan and Zaifu Yang are sincerely acknowledged. The first author thanks CentER and NWO for financial support and hospitality at Tilburg University. 
Open Access This article is distributed under the terms of the Creative Commons Attribution Noncommercial License which permits any noncommercial use, distribution, and reproduction in any medium, provided the original author(s) and source are credited.

\section{References}

Borm P, Owen G, Tijs S (1992) On the position value for communication situations. SIAM J Discrete Math 5:305-320

Demange G (1994) Intermediate preferences and stable coalition structures. J Math Econ 23:45-58

Demange G (2004) On group stability in hierarchies and networks. J Polit Econ 112:754-778

Herings PJJ, van der Laan G, Talman D (2008) The average tree solution for cycle-free graph games. Games Econ Behav 62:77-92

Kaneko M, Wooders M (1982) Cores of partitioning games. Math Social Sci 3:313-327

Le Breton M, Owen G, Weber S (1992) Strongly balanced cooperative games. Int J Game Theory 20: $419-427$

Myerson R (1977) Graphs and cooperation in games. Math Oper Res 2:225-229

Myerson R (1980) Conference structures and fair allocation rules. Int J Game Theory 9:169-182

Shapley L (1953) A value for $n$-person games. In: Kuhn H, Tucker A (eds) Contributions to the theory of games II. Princeton University Press, Princeton pp 307-317 